\title{
THERMOMECHANICAL BEHAVIOR OF MICRON SCALE SOLDER JOINTS: AN EXPERIMENTAL OBSERVATION
}

\author{
Zhao, Y. ${ }^{1}$, Basaran ${ }^{*}$, C. $^{2}$, Cartwright, A. ${ }^{3}$, Dishongh, T. ${ }^{4}$ \\ ${ }^{1}$ Ph.D. candidate, ${ }^{2}$ Assoc. Prof. and Director, ${ }^{3}$ Assist. Prof., \\ Electronic Packaging Laboratory, 212 Ketter Hall, SUNY at Buffalo, 'NY 14260, USA \\ ${ }^{4}$ Senior Engineer, Intel Corp., Hillsboro, OR, USA
}

\begin{abstract}
The study of solder joint reliability and integrity is a significant issue in electronic packaging. Solder joints are subjected to cyclic strain due to thermal expansion mismatch between the bonded layers, as a result they undergo elastic and inelastic deformations, and finally experience microcracking and damage. There is very little test data available in the literature about thermomechanical response of actual micron scale solder joints in Ball Grid Array (BGA) products. In this study, thermal cycling of a BGA package between $-55^{\circ} \mathrm{C}$ and $125^{\circ} \mathrm{C}$ is conducted by using a large capacity Super AGREE thermal chamber. High sensitivity Moiré Interferometry is used for measuring the irreversible strain after certain number of cycles. A novel specimen preparation technique allowed us to measure irreversible strain accumulation up to 20 thermal cycles. The technique used casts a new look in the application of Moiré Interferometry method. Previously reported similar studies in the literature included one thermal cycle only. This new technique allows recording plastic deformations up to twenty cycles.
\end{abstract}

\section{INTRODUCTION}

Microelectronic devices typically are multilayered structures, and each layer has different material properties such as coefficient of thermal expansion (CTE), elastic moduli, hardening coefficients, etc. The solder joints in electronic packaging usually act as mechanical bonds as well as electrical interconnections. Thermo-mechanical fatigue is the deformation condition that arises when structural material encounters temperature fluctuations while in service. The fluctuations result in induced cyclical strains in the solder joints.

The US Air Force estimated that vibration and shock cause 20 percent of the mechanical failures in airborne electronics. Interestingly, the remaining 80 percent of mechanical failures relate to thermal

"Corresponding author cjb@eng.buffalo.edu 
stresses induced by high thermal gradients, high CTE mismatch, according to Markstein (1987).

The state-of-the-art thermomechanical fatigue testing methodologies were categorized into four major types by Frear (1990): The thermal cycling of actual microelectronic components, thermocycling of simplified test specimens, thermomechanical fatigue of bulk solder joints, and thermomechanical fatigue of solder joints. Firstly, it has been proven that the material properties of bulk specimen are very different from real micron scale solder joints due to the size effect, Bonda and Noyan (1996). Secondly, the conventional thermal cycling of solder joints is usually used for developing Coffin-Manson type empirical life prediction curves. The damage accumulation is checked during cycling, and final failure is monitored by visual checking of crack initiation and propagation, or by measuring the electrical impedance of the solder joints. However, these measurements do not provide detailed information on irreversible strain accumulation and material property degradation, and are also difficult to be used in developing suitable constitutive models for further computer simulation of fatigue life prediction. Due to difficulty in recording and measuring irreversible deformations on actual real-life BGA solder joints, there is very little data reported in the literature. Until now most of the published experimental work reported recording irreversible strains for just one thermal cycle only.

This paper reports the development of a new technique for measuring fatigue damage accumulation by using Moiré Interferometry for monitoring irreversible strain accumulation during thermal cycling on real life electronic packaging for many cycles.

Moiré Interferometry (MI) has been applied effectively to electronic packaging devices. The application of this technique can be categorized in three types: First, direct measurement of whole-field displacement under thermal mechanical loading, Han and Guo $(1995,1996)$, Verına et al. (1998), to name a few. The second type is hybrid experimental/ computational analysis of stress and strain, such as Zhu and Liu (1997), Wang et al. (1998). Finally, determination of material properties using MI. For example, Guo (1995) applied a uniform thermal gradient to obtain material CTE, and Post et al. (1989) applied rail-shear loading to the specimen to determine shear moduli of cross-ply laminates. Most of the thermal tests applied temperature gradient monotonically, and almost all of these experiments were performed for understanding the deformation/failure mechanism, and stress-strain response by combining with the finite element analysis. Han (1997) published a pioneering work in which he reported recording plastic strains up to four cycles on two-phase solder column interconnections. The experimental technique developed here is a new application area of Moiré Interferometry which allows recording irreversible strains (plastic and creep) up to 20 cycles.

\section{MOIRE INTERFEROMETRY}

The Moiré Interferometry (MI) is used here to record the irreversible deformations during thermal cycling. The advantage of this method is that it provides a whole-field view of the tested sample, and it also yields submicron high resolution for measuring the small deformation of a small area. One has to 
note that small deformation does not infer small strain. In the case of solder joints under thermal mechanical cycling, it always corresponds to high strain due to the small dimension of solder joints.

A detailed description of MI can be found in Post et al. (1994). In short, MI uses two coherent beams of light to generate an interferometric fringe pattern which carries the information of in-plane deformation of the object surface that is illuminated by the coherent light. A thin layer of epoxy is applied to the specimen surface to replicate optical diffraction grating on the surface. The diffraction grating deforms with the specimen surface as the specimen is loaded, and the diffracted light records every detail of the deformed lines of the grating and thus records the deformation of specimen surface. The fringe pattern can be related to in-plane deformation quantitatively as given by Post et al (1994):

$$
\begin{aligned}
& U:=\frac{N x}{f} \\
& V=\frac{N y}{f}
\end{aligned}
$$

where $U$ is the displacement in $x$ direction; $V$ is the displacement in $y$ direction; $f=2 f s$, $f s$ is the frequency of specimen diffraction grating, in this study, $f s=1200$ lines $/ \mathrm{mm}$; $\mathrm{Nx}$ is horizontal fringe order, and $\mathrm{Ny}$ is the vertical fringe order.

Once the displacement data are available the total strains are computed by differentiation of the displacement distributions with respect to the two basic directions: horizontal $(x)$ and vertical $(y)$. The strains are given by,

$$
\begin{gathered}
\varepsilon_{:}=\frac{\partial U}{\partial x}=\frac{1}{f}\left[\frac{\partial N_{x}}{\partial x}\right] \\
\varepsilon_{y}=\frac{\partial V}{\partial y}=\frac{1}{f}\left[\frac{\partial N_{y}}{\partial y}\right] \\
\gamma_{x y}=\frac{\partial U}{\partial y}+\frac{\partial V}{\partial x}=\frac{1}{f}\left[\frac{\partial N_{x}}{\partial y}+\frac{\partial N_{y}}{\partial x}\right]
\end{gathered}
$$

\section{THE BASIC SETUP OF MOIRE INTERFEROMETRY}

The Moiré Interferometry system shown in Figure 1 is composed of a He-Ne laser as coherent light source, a micro-objective to expand the beam, a spatial filter to reduce the high frequency noise in the light, a big lens to collimate the beam, the adjustable multi-mirror mounting system, the specimen 


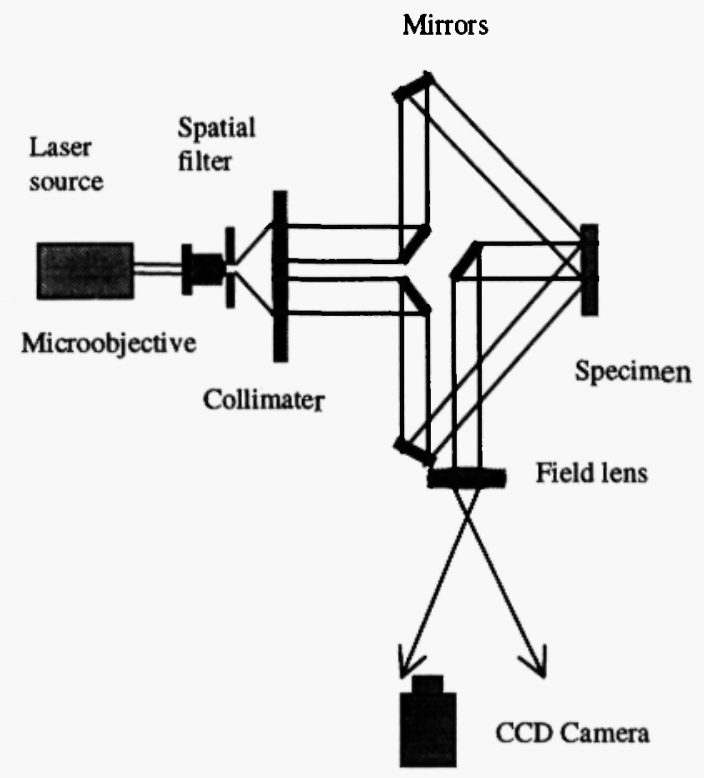

Fig. 1: Optical setup of Moiré Interferometry

fixture, and the imaging system. There are other configurations used for MI in the literature, see Post $e t$ al. (1994). The multi-mirror mount is the heart of the MI system used in this project. It actually generates two coherent laser beams in both horizontal and vertical directions individually from the same coherent light source. Each two beams are in the same plane, and they generate an interferometric fringe pattern. As shown in Figure 2, the multi-mirror mounting system consists of a highly sensitive precision frame with a central mirror mount which supports the inner four mirrors. Four outside mirrors in the multi-mirror system are mounted on the adjustable mirror mounts individually.

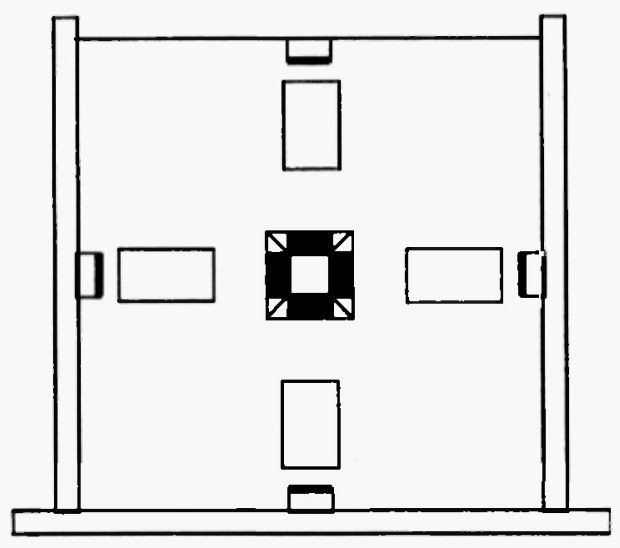

Fig. 2: Precision Multimirror Mount 


\section{IMAGING SYSTEM}

Two imaging systems have been used to capture different types of images as shown in Figure 3.

The first imaging system is used to capture the enlarged image of individual solder joints to observe detailed local deformation. A plane mirror (Ml) and a field lens (Lensl) are the main components in system 1 . The plane mirror is to direct the imaging beam to the CCD camera, and field lens is to collect the beam and enlarge the image. The CCD camera was mounted on a 3D mechanical translator, so that its position can be adjusted precisely. Especially, in the horizontal direction, enough free path is provided so that the CCD camera can capture each solder joint of interest by moving along the free path. The field lens needs to be big so that the imaging beams can be as centered as possible to avoid image distortion and to obtain the best quality of the image.

The second imaging system is used to capture the image of the entire specimen to give a global view of the deformations in all solder joints. For this purpose, another short focal-length lens (Lens2) is added to focus the entire image so that the whole image just fits in the area array of the photo detectors of the CCD camera. The light-intensity is reduced in this case to prevent saturation of the CCD. Saturation turns the image into a field of white noise.

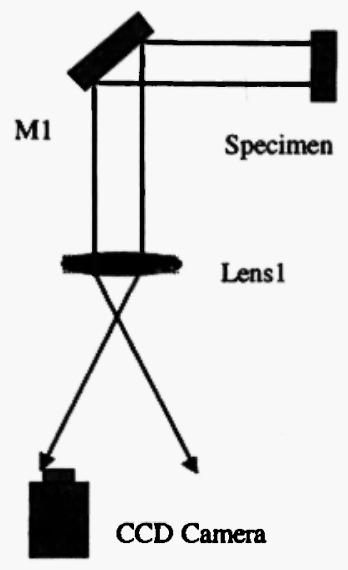

Fig. 3(a): Imaging System 1

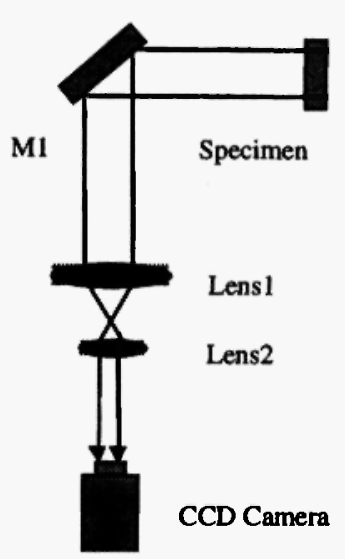

Fig. 3(b): Imaging System 2

\section{TESTING PROCEDURE}

The BGA package studied here is for a high-speed new generation CPU processor. The specimen cross-section is shown in Figure 4. It has a multilayered structure, with three major layers connected by two layers of BGA solder joints. All the solder joints are $\mathrm{Sn} 63 / \mathrm{Pb} 37$ eutectic solder alloy. The solder joints of interest are at the bottom layer which bonds two layers with significantly different coefficients 


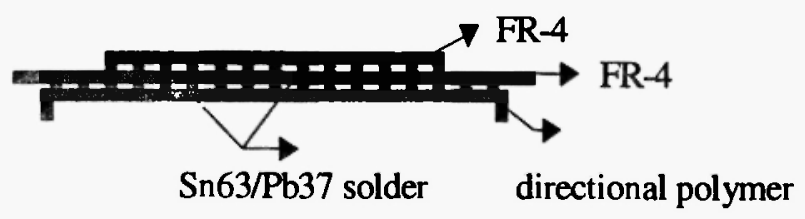

Fig. 4: Cross-section of the specimen

of thermal expansion (CTE) - the FR4 layer and the long chain directional polymer layer. These solder joints have been observed to be the most vulnerable interconnections of the entire device. The CTE of the polymer is about 5 orders bigger than the FR4. There are tiny thin copper metallization layers inserted in the polymer, which actually bond to the solder joints.

The specimen was cut by a high precision diamond wheel saw right through the center of the solder joints of interest. The exposed cross section was then polished by using abrasive papers with different grain sizes, i.e. $\# 400, \# 800$, and $\# 1200$. The surface was then cleaned and dried thoroughly and ready for diffraction grating replication. Probably one of the most important parts of this project was selection of the epoxy. A proper type of epoxy was chosen after many trial and errors to make sure that the specimen grating would stand the peak cycling temperature for many cycles. The epoxy was also chosen to have a very low viscosity to achieve a very thin layer as it was spread out on the specimen surface to replicate the grating. The two-component epoxy was fully mixed and sent to a vacuum chamber for degas. After degassing, the epoxy was spread carefully on the specimen surface, and a master grating was stamped onto the surface. The specimen and master grating pair was kept tightly compressed against each other until the end of the epoxy curing period. The specimen was then separated from the master grating with the replicated diffraction grating on its surface. It is important to control the degas procedure to be completed in a certain amount of time so that enough time is left for replication before the epoxy working life limit is reached. The grating used in this project is 1200 lines $/ \mathrm{mm}$.

The specimen was thermally cycled in a high capacity Super AGREE environmental chamber between $-55^{\circ} \mathrm{C}$ and $125^{\circ} \mathrm{C}$. The temperature changing rate was $20^{\circ} \mathrm{C} / \mathrm{min}$, the holding time at each extreme end was $12 \mathrm{~min}$, and the period of one cycle was $42 \mathrm{~min}$. The temperature history is shown in Figure 5. Before cycling, the specimen was used to initialize the interferometry system, i.e. to get a null field of the image which represents a zero deformation field. The whole optical system was then isolated and kept untouched during the testing procedure. A special high precision device was developed to register the position of the specimen, so that each time the specimen was taken away for thermal cycling, we were able to put it right back at the same position. Another fixture was designed to secure the specimen through the testing and to keep the boundary condition of the specimen unchanged through the entire testing procedure. The technique developed here guaranteed that the temperature gradient was the only loading, and the measured displacement truly represented the irreversible plastic 


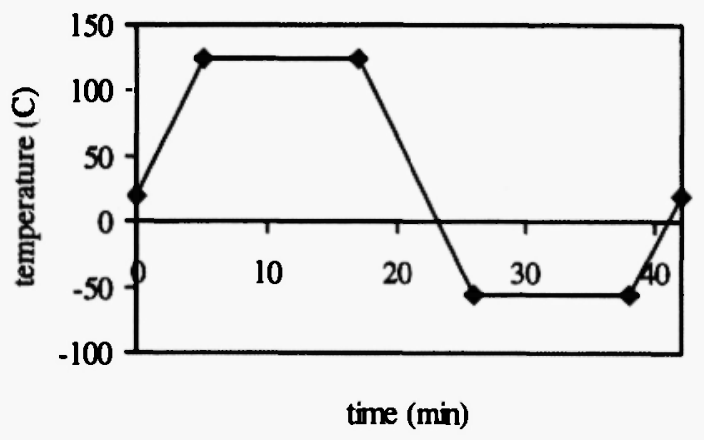

Fig. 5: Temperature history

deformation of the specimen.

The specimen was exposed to thermal cycling for certain cycles, then it was taken out to measure the irreversible deformation. It was then put back to the chamber for further cycling. For each measurement, the total irreversible deformation was recorded and average strain at each solder joint was calculated. At the same time, the damage propagation was monitored by examining the fringe pattern.

\section{DISCUSSION OF THE RESULTS}

The whole field images of half of the specimen are shown in Figure 6 and Figure 7 for the deformation after one cycle, and in Figure 8 and Figure 9 for the deformation after 10 cycles. It is easy to see that normal deformation in both $\mathrm{x}$ and $\mathrm{y}$ direction is ignorrable while shear deformation dominates. The source of fatigue during thermal cycling is cycling shear strain, and the relevant failure mechanism is creep fatigue in shear. It is also obvious that shear strain gradually increases from the center solder joint (solder 10) to the edge solder joint (solderl), counting from the free edge to the center. The irreversible shear deformations of certain solders were also recorded to track the plastic deformation accumulation during each thermal cycling. The fringe patterns of solder joint 3 after certain cycles are shown in Figure $10(\mathrm{a}-1)$ as an example. Deformation is reasonably homogeneous in each solder joint.

Figure 11 shows the average accumulative irreversible shear strain distribution of the solder joint layer at $1^{\text {st }}, 2^{\text {nd }}, 4^{\text {th }}, 6^{\text {th }}, 10^{\text {th }}, 14^{\text {th }}$ and $20^{\text {th }}$ cycles. The shear distribution diagram indicates that outside solder joint experiences the biggest shear loading, therefore the failure of the solder joint layer might start from the most outer solder joint if the solder joints are of the same quality.

The calculated average irreversible shear strain versus number of thermal cycles is plotted in Figure 12 for solder 1 , solder 2 , solder 3 , solder 4 , solder 5 and solder6. It can be seen that the biggest irreversible 


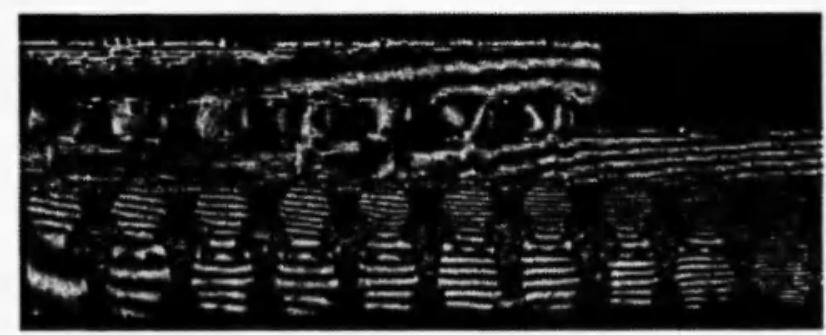

Fig. 6: The $U$ field of half of the specimen after one thermal cycle.

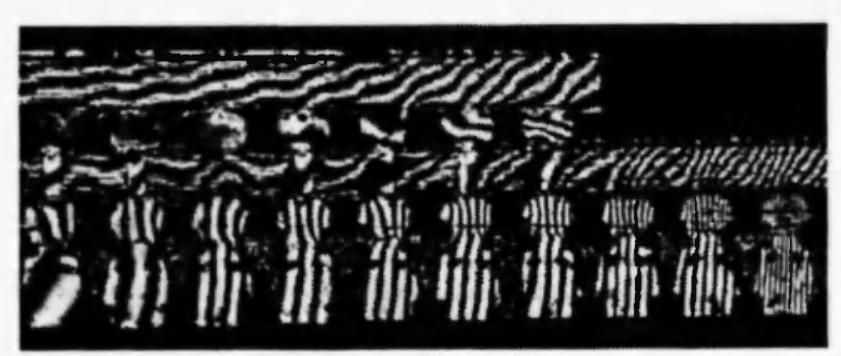

Fig. 7: The $V$ field of half of the specimen after one thermal cycle.

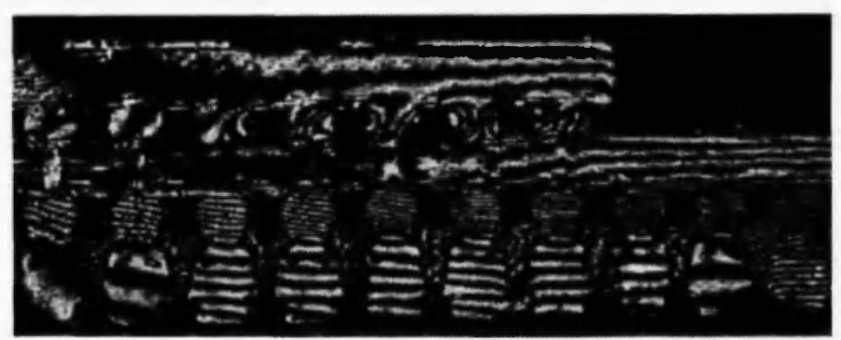

Fig. 8: The $U$ field of half of the specimen after 10 thermal cycles.

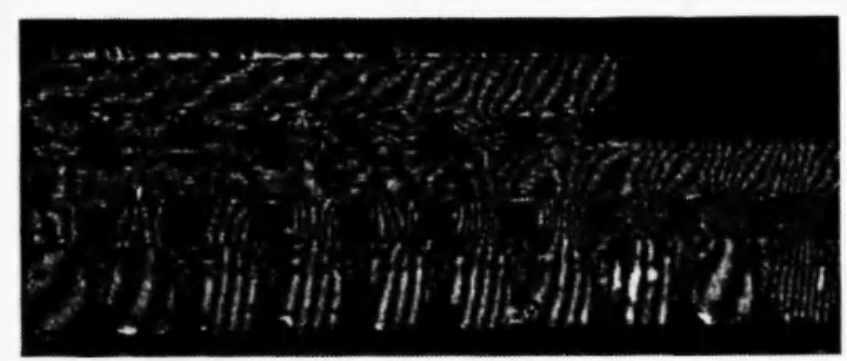

Fig. 9: The $\mathrm{V}$ field of half of the specimen after 10 thermal cycles.

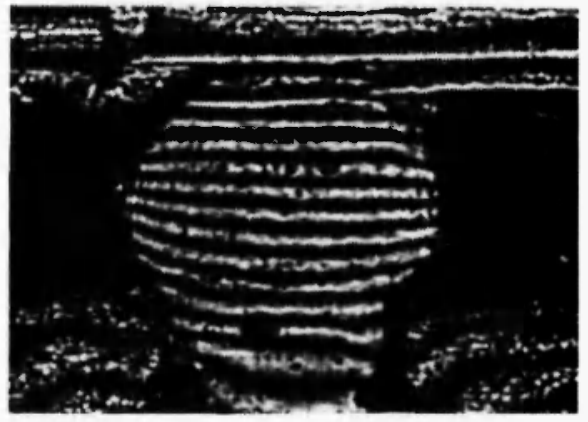

Fig. 10(a): U field of Solder 3 after 1 thermal cycle.

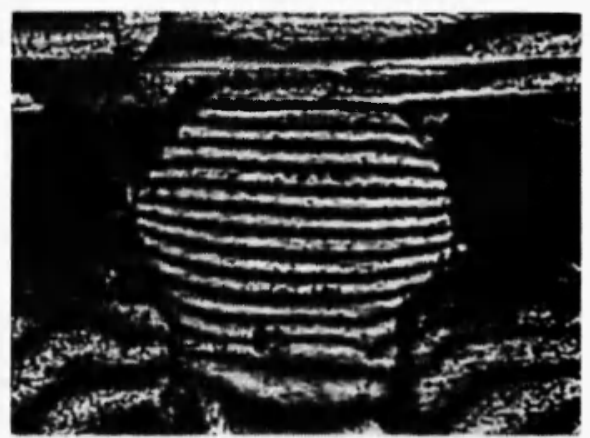

Fig. 10(b): U field of Solder 3 after 2 thermal cycles.

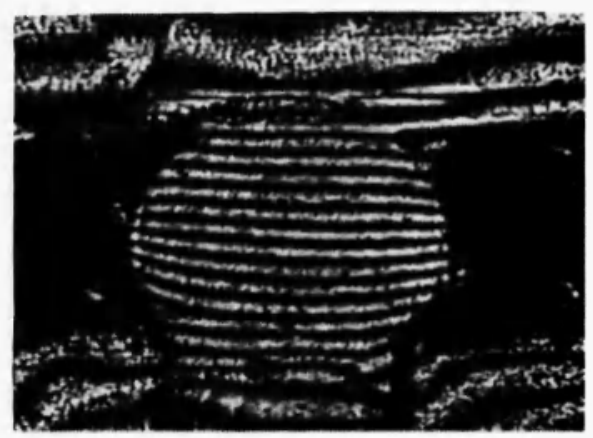

Fig. 10(c): $U$ field of Solder 3 after 4 thermal cycles.

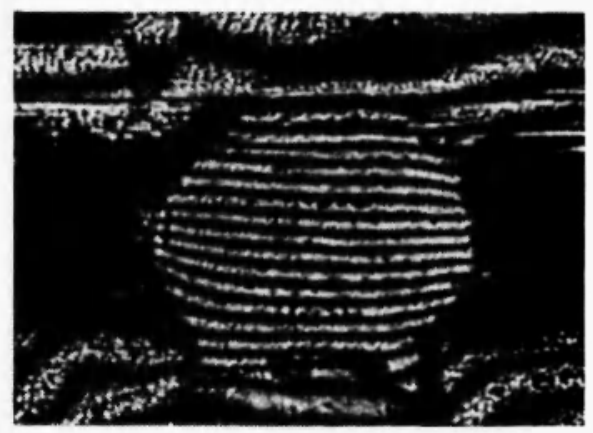

Fig. 10(d): U field of Solder 3 after 6 thermal cycles. 
Zhao, Y. et al.

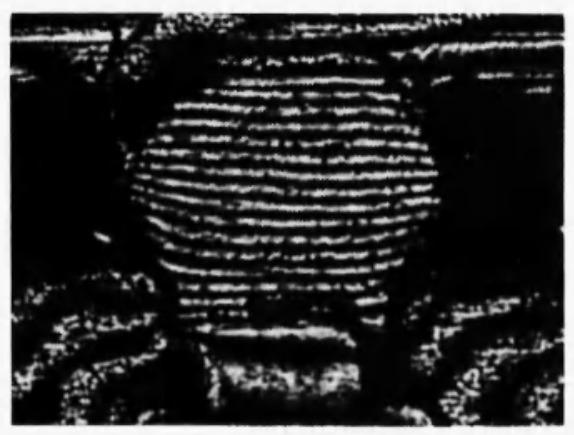

Fig. 10(e): $U$ field of Solder 3 after 10 thermal cycles.

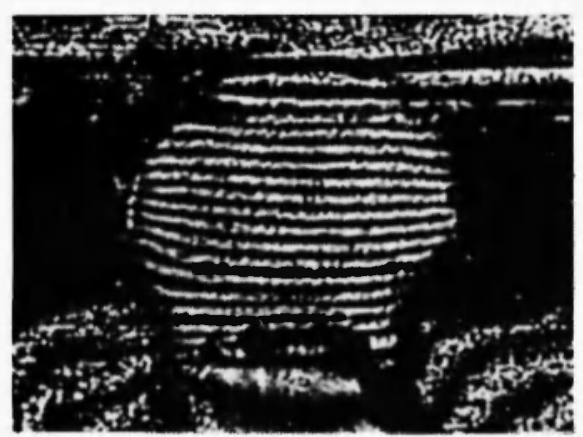

Fig. 10(f): U field of Solder 3 after 14 thermal cycles.

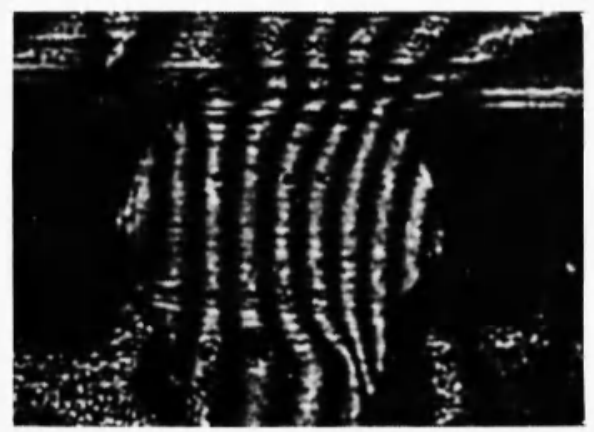

Fig. 10(g): $V$ field of Solder 3 after 1 thermal cycle.

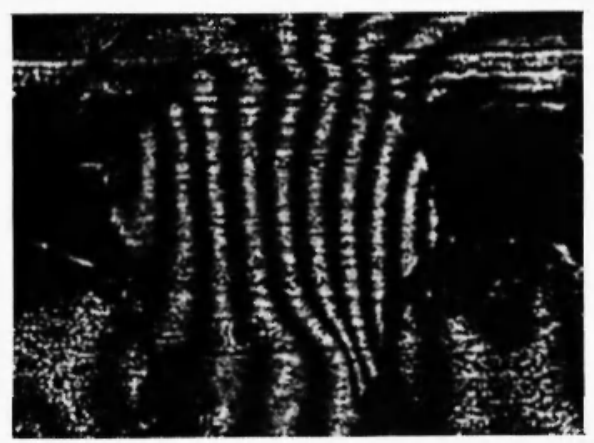

Fig. 10(h): $V$ field of Solder 3 after 2 thermal cycles.
Journal of the Mechanical Behavior of Materials

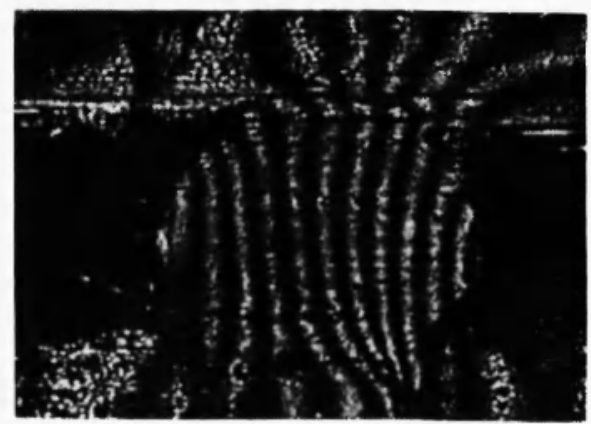

Fig. 10(i): $V$ field of Solder 3 after 4 thermal cycles.

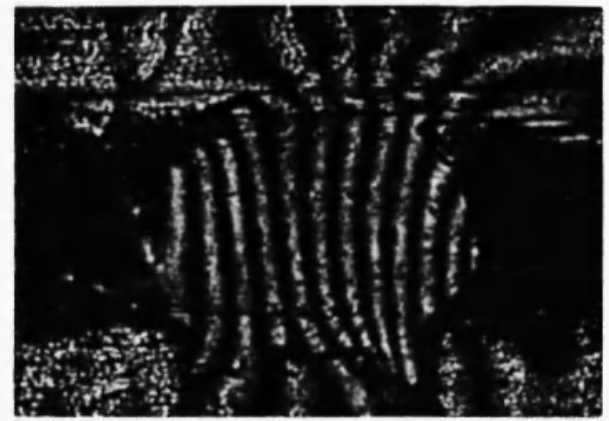

Fig. 10(j): V field of Solder 3 after 6 thermal cycles.

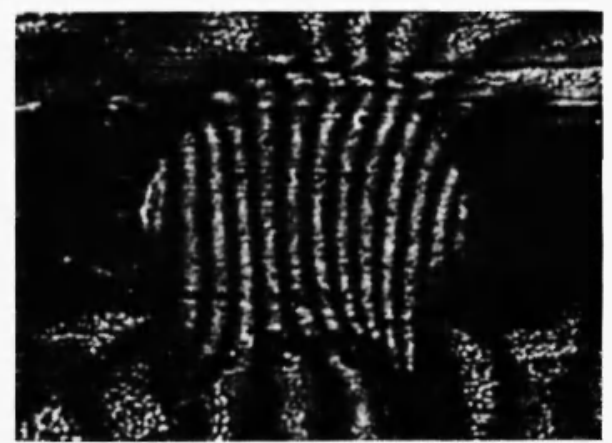

Fig. 10(k): $\mathrm{V}$ field of Solder 3 after 10 thermal cycles.

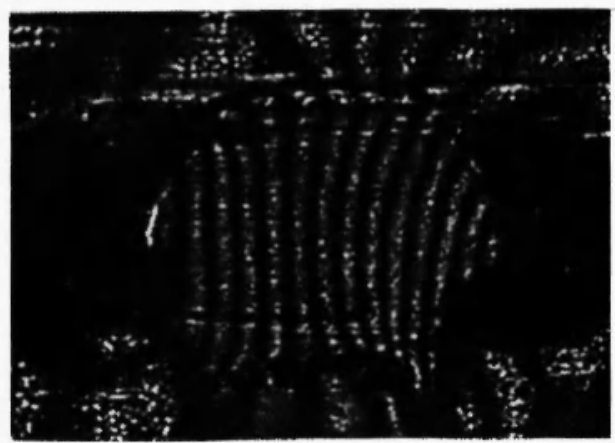

Fig. 10(I): $V$ field of Solder 3 after 14 thermal cycles. 


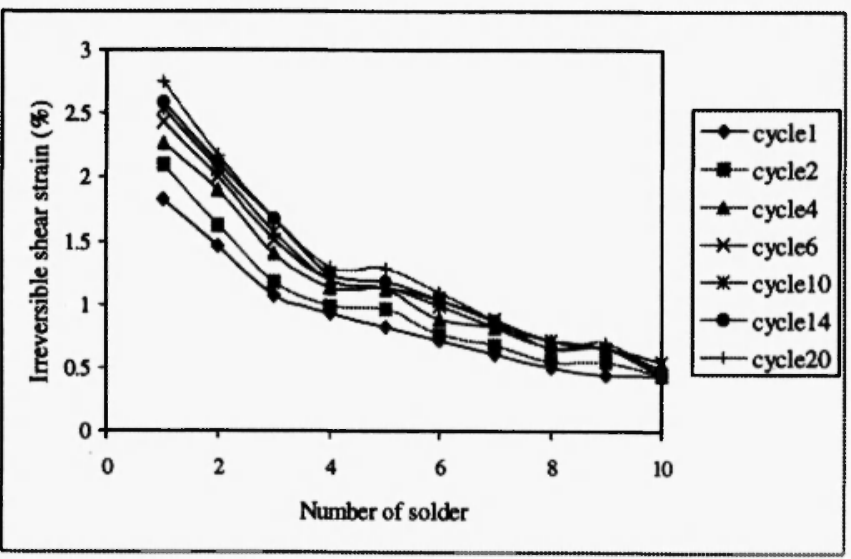

Fig. 11: Irreversible shear strain distribution

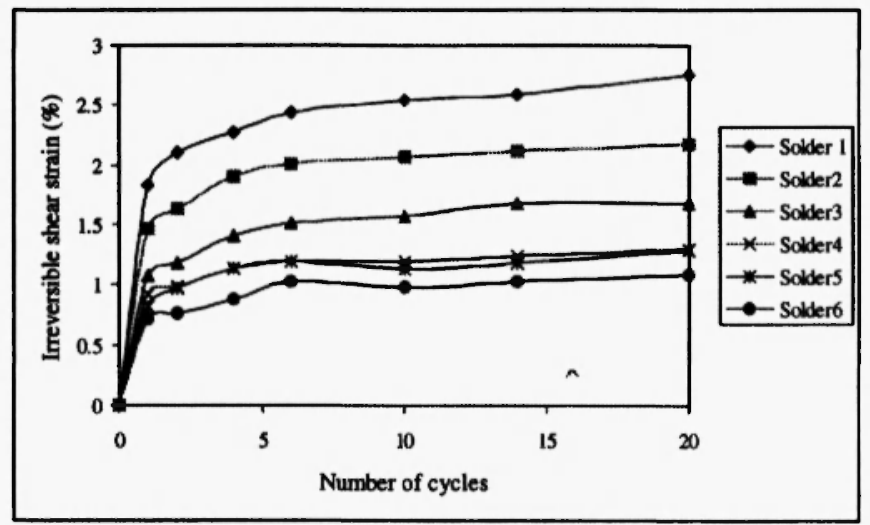

Fig. 12: Irreversible shear strain accumulation

shear strain is induced in the first cycle. As the number of cycles increase, the additional plastic deformation in each cycle decreases. Virtually every solder joint presents this characteristic. This interesting phenomenon actually contains very useful information and requires special consideration about thermal fatigue. This observation shows that using Miners rule for fatigue life calculation is not accurate. This latter finding is also in line with recent findings reported by Basaran and Chandaroy, (1998, 1999), where authors analytically show that damage accumulation is not linear. First, thermal cycling process is not a simple creep or stress relaxation process but a complex combination of both. In each cycle, thermal stresses are not the same even at the same temperature level. Furthermore, since the eutectic solder joints are at a high homologous temperature during testing, as they ordinarily are under service conditions, the microstructure may evolve. Microstructural change, such as grain coarsening, can significantly affect the material properties, such as creep mechanism, shear strength, and stress 
relaxation behavior. Analytic models that attempt to predict joint behavior should be viewed with skepticism if they do not incorporate the effect of microstructural evolution, Morris and Reynolds (1991), Basaran and Chandaroy (1998).

Joint failure can be examined by checking the fringe pattern. Since the fringe pattern represents the contour of the in-plane deformation, any discontinuity in the fringe pattern may imply the initiation of a crack if the specimen grating is of good quality. Careful study of Figure 10a to 1 shows locations of discontinuities in fringe pattern.

\section{CONCLUSIONS}

Thermal cycling was conducted on a multilayered new generation CPU device with $\mathrm{Sn} 63 / \mathrm{Pb} 37$ eutectic solder (BGA) as the bonding layer. Moiré Interferometry technique has been successfully applied to measure the whole field irreversible deformation for 20 cycles. It is observed that the accumulation of irreversible strain, which is usually used as a damage metric, is not linear. Global view of the whole package deformation is obtained as well as local average deformation on individual solders. Both U field and V field of deformation are analyzed to extract the strain values. Shear strain is the major cause of fatigue failure during thermal cycling.

The Moiré Interferometry method is an effective method for recording plastic deformation accumulation, though important attention has to be paid when designing the testing setup to insure that no additional strain is induced during each recording. This technique provides a useful method to test the solder joints in real life packaging. Most importantly, testing actual packages allows accounting for the material size effect.

Improving the technique presented in this paper, it should be possible to record irreversible deformations up to a much higher number of thermal cycles.

\section{ACKNOWLEDGMENTS}

This research project is sponsored by the Department of Defense Office of Naval Research Young Investigator Award to Dr. C. Basaran. Helpful discussions with Dr. Roshdy Barsoum, Director of Solid Mechanics Program at ONR are gratefully acknowledged. 


\section{REFERENCE}

Basaran, C. and Chandaroy, R., "Mechanics of Pb40/Sn60 Near Eutectic Solder Alloys Subjected to Vibrations", Applied Mathematical Modeling, 22, 601-627 (1998).

Basaran, C. and Chandaroy, R., "Using FEM for Simulation of Reliability Tests on Solder Joints in Microelectronic Packaging", Computer \& Structures, in print, 1999.

Bonda, N.R., and Noyan, I.C., "Effect of the Specimen Size In Predicting the Mechanical Properties of PbSn Solder Alloys," Trans. Comp. Pack. And Man., Tech. A 19 (2) (Year?)(page nos.?)

Frear, D.R., Jones, W.B. and Kinsman, K.R. Solder Mechanics, State of the Art Assessment, Santa Fe, New Mexico, 1990.

Guo, Y. "Experimental Determination of Effective Coefficients of Thermal Expansion in Electronic Packaging", EEP-Vol.10-2, Advances In Electronic Packaging, ASME 1995, p. 1253-1264.

Han, B. and Guo, Y. "Thermal Deformation Analysis of Various Electronic Packaging Products by Moiré and Microscopic Moire Interferometry", Journal of Electronic Packaging, 117, 185-191 (1995).

Han, B. and Guo, Y. "Determination of an Effective Coefficient of Thermal Expansion of Electronic Packaging Components: A Whole-Field Approach", IEEE Transactions on Components, Packaging, and Manufacturing Technology-Part A, 19 (2), 240-247 (1996).

Han, B. "Deformation Mechanism of Two-Phase Solder Column Interconnections Under Highly Accelerated Thermal Cycling Condition: An Experimental Study", Journal of Electronic Packaging, 119, 189-196 (1997).

Markstein, H. W. "Designing Electronics for High Vibration and Shock", Electronic Packaging \& Production, 40-43 (April, 1987).

Morris, Jr. J.W. and Reynolds, H.L. "The Influence of Microstructure on the Mechanics of Eutectic Solders", EEP Vol.19-2, Advances in Electronic Packaging, ASME, 1991, p.1529.

Post, D., Han, B. and Ifju, P. High Sensitivity Moire, Springer-Verlag, 1994.

Post, D., Dai, F.L. , Guo, Y. and Ifju,, P. "Interlaminar Shear Moduli of Cross Laminates: An Experimental Analysis", Journal of Composite Materials, 23, 265-279 (1989).

Verma, K., Kunthong, P. and Han, B. "Thermo-Mechanical Strains of Flip-Chip Solder Bumps on Organic PCB Substrate", ASME International Mechanical Congress \& Exposition, Anahein, California, Nov. 15-20, 1998.

Wang, J., Qian, Z., Zou, D, and Liu, S. "Creep Behavior of a Flip-Chip Package by Both FEM Modeling and Real Time Moire Interferometry", Journal of Electronic Packaging, 120, 179-185 (1998).

Zhu, J. and Liu, S., "Thermal Deformation And Stress Analysis Of A Column Grid Array Package by Hybrid Moire/FEM Method", ASME International Mechanical Engineering Congress \& Exposition, Dallas, Texas, Nov. 16-21, 1997. 